\title{
Comparison of solar activity during last two minima on turn of Activity Cycles 22/23 and 23/24
}

\section{Magdalena Gryciuk ${ }^{1}$, Szymon Gburek ${ }^{1}$, Marek Siarkowski ${ }^{1}$, Piotr Podgorski ${ }^{1}$, Janusz Sylwester $^{1}$ and Frantisek Farnik ${ }^{2}$}

\author{
${ }^{1}$ Space Research Centre, Polish Academy of Sciences, 51-622, \\ Kopernika 11, Wroclaw, Poland, e-mail: mg@cbk.pan.wroc.pl \\ ${ }^{2}$ Astronomical Institute, Ondrejov Observatory, Czech Republic
}

\begin{abstract}
The subject of our work is the review and comparison of solar activity during the last two solar minima that occurred between recent activity cycles. We use the soft X-ray global solar corona observations covering the two nine-months long time intervals in 1997/98 and 2009. Data from RF15-I multichannel photometer are used for the penultimate minimum. For the last unusually deep and prolonged solar activity minimum in 2009 the data from SphinX spectrophotometer are used. Comparison of measurements from both minima takes place in the overlapping energy range $2-15 \mathrm{keV}$. We focus on the active region formation, evolution and flaring productivity during respective minima.
\end{abstract}

Keywords. Sun: activity, Sun: corona, Sun: X-rays, gamma rays

\section{Introduction}

Two 281-days long time periods were selected for the present comparison analysis. Both periods cover similar parts of two consecutive solar cycles minima of activity. Their start times were found as equidistant to start times of respective solar cycles. The first time interval covered by RF15-I (Sylwester et al. 2000) measurements consist of data from 02 July 1997 to 10 April 1998 while the second period of SphinX (Sylwester et al. 2008; Gburek et al. 2011) measurements extends from 20 February to 29 November 2009. The duration of both periods equals to active lifetime of the CORONAS-Photon mission.

\section{Observations}

In Figure 1 there are light curves of RF15-I and SphinX which were obtained by integrating spectra over overlapping energy ranges of the instruments. Active regions (AR) related characteristic features and flares can be recognized on the light curves in both cases. For the comparison the solar flux observed by GOES in energy range $1-8 \AA$ during analysed time periods are shown. A very good match is evident in both cases. For the second time interval it is clearly seen that SphinX sensitivity is much superior in respect to GOES. Right panels of Figure 1 present ARs trajectories on the visible solar hemisphere during analysed time periods. Times and locations of regions on solar disc were taken from Joint USAF/NOAA Solar Region Summary Reports.

In Table 1 we present comparison of activity indicators for both analysed time intervals on turn of solar cycles of activity $22 / 23$ and $23 / 24$. Number of days when sunspots were observed was obtained based on data provided by NOAA. In the second column we compare number of flares with GOES class above B1.0. We used GOES flares catalog for this statistics. Last column contains the number of ARs visible on solar disc. 

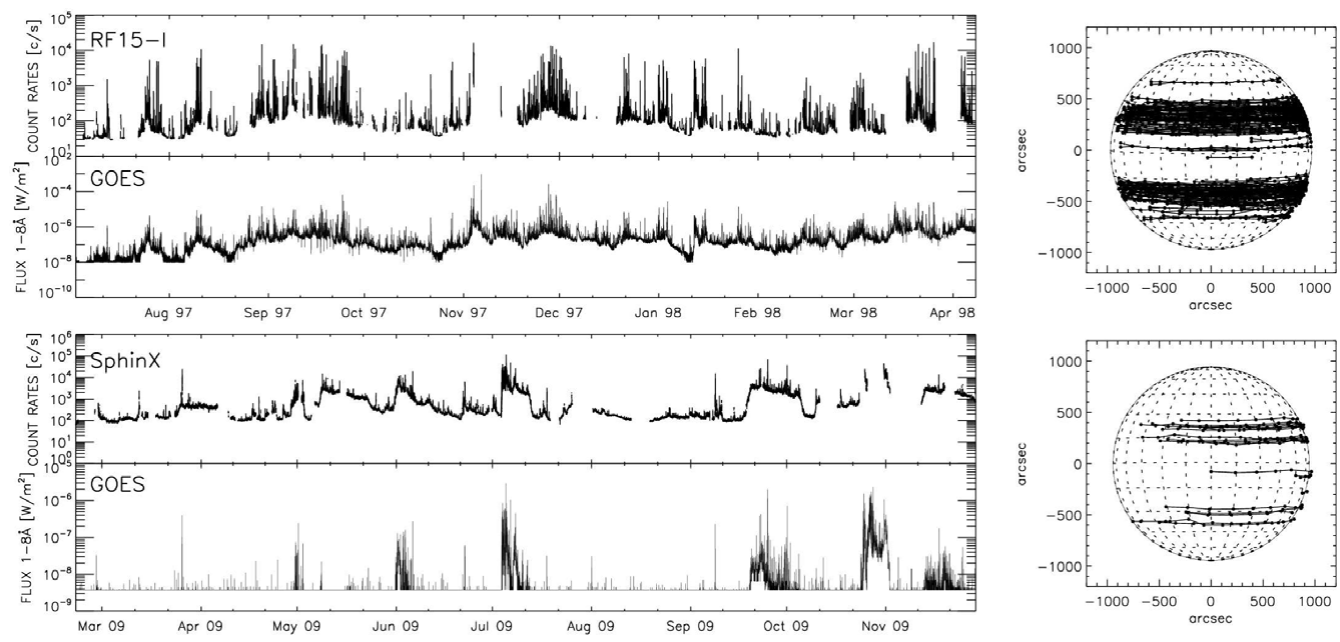

Figure 1. Top-left: RF15-I count rates observed during period from 02 July 1997 to 10 April 1998. Bottom-left: SphinX count rates covering period from 20 February to 29 November 2009. Below respective plots of GOES fluxes in 1-8 A spectral band are shown. Right plots: Trajectories of ARs visible on the solar disc for respective time periods.

\begin{tabular}{|l|c|c|c|}
\hline & $\begin{array}{c}\text { Number of } \\
\text { Days with Sunspots }\end{array}$ & $\begin{array}{c}\text { Number of Flares over } \\
\text { B1.0 GOES Class }\end{array}$ & $\begin{array}{c}\text { Number of ARs } \\
\text { on Solar Disc }\end{array}$ \\
\hline 02 Jul 1997 - 10 Apr 1998 (minimum 22/23) & 265 (94\% of time) & 1465 & $\mid$ \\
\hline 20 Feb 2009-29 Nov 2009 (minimum 23/24) & $71(24 \%$ of time) & 131 & 150 \\
\hline
\end{tabular}

Table 1. Comparison of solar activity indicators for two analysed time intervals.

\section{Conclusions}

Comparison of magnetic activity indicators shows that the active Sun atmosphere displayed substantially different conditions during analysed periods. Number of days with spots on solar disc was about four times smaller in the second period than in the first one. Number of flares above GOES B1.0 class decreased approximately ten-fold and the number of ARs dropped by a factor of $\sim 7$ as it is seen from Table 1 . The ARs paths of preceding minimum covered much wider latitudinal belts in comparison with the recent minimum. Formation of near-equator ARs during recent minimum was almost suppressed. This supports the common opinion of unusual character of the recent activity minimum (Dikpati 2011).

Acknowledgements: We acknowledge financial support from the Polish National Science Centre grant 2011/01/B/ST9/05861 and from the European Commision Seventh Framework Programme under the grant agreement No. 284461 (eHEROES project).

\section{References}

Dikpati, M. 2011, Space Sci. Revs, in press

Gburek, S., Siarkowski, M., Kepa A., Sylwester, J. et al.. 2011, Solar System Research, 45, 182 Sylwester, J., Farnik, F., Likin, O., Kordylewski, et al. 2000, Solar Phys., 197, 337

Sylwester, J., Kuzin, S., Kotov, Yu. D., Farnik, F., \& Reale, F. 2008, J. Astrophys. Astron., 29, 339 